\title{
Capacidade cardiovascular e composição corporal relacionadas à hidrocinesioterapia nos períodos críticos da adaptação materna à gestação
}

\section{Cardiovascular volume and body composition in relation to the use of hydrokinetic therapy during the critical periods of maternal adaptation to pregnancy.}

Autora: Tânia Terezinha Scudeller Prevedel

Orientadora: Profa. Dra. Iracema de Mattos Paranhos Calderon

Tese de Doutorado apresentada ao Curso de Pós-Graduação em Ginecologia, Obstetrícia e Mastologia; Área de concentração: Obstetrícia, da Faculdade de Medicina de Botucatu/SP - UNESP, em 20 de maio de 2005.

Objetivo: estudar a capacidade cardiovascular e a composição corporal materna relacionadas à prática de exercício aquático terapêutico (hidrocinesioterapia) nos períodos de sobrecarga hemodinâmica e de adaptação metabólica à gestação. Métodos: ensaio clínico controlado e não-cego, com 41 gestantes saudáveis, randomizadas em dois grupos - Controle e Hidrocinesioterapia. Foram avaliados a capacidade cardiovascular $\left(\mathrm{VO}_{2}\right.$ máx, débito cardíaco [DC], volume sistólico [VS], pressão arterial média), o desempenho físico materno, a composição corporal (peso, massa magra $[\mathrm{MM}]$, gordura absoluta [GA] e relativa [GR]), a antropometria materna (dobras cutâneas subescapular [DCSE], supra-iliaca [DCSI] e da coxa [DCC], circunferências abdominal [CA] e do quadril [CQ]), a altura uterina [AU] e o prognóstico neonatal. As médias foram avaliadas pelo teste $t$, dependente e independente, e as proporções, pelo teste do qui-quadrado, com $\mathrm{p}<0,05$. Resultados: as variáveis de controle atestaram a homogeneidade dos gru- pos. A hidrocinesioterapia se relacionou à manutenção do $\mathrm{VO}_{2}$ max, aumento do VS e DC e melhor desempenho nos testes de esforço do terceiro trimestre. O peso, a MM e a GA foram progressivos nos dois grupos, as DCSE e DCC aumentaram no Controle e mantiveram-se no grupo Hidrocinesioterapia, sem diferença na evolução da DCSI. Apesar da intervenção, houve incremento de CA, CQ e AU. Os resultados neonatais foram semelhantes nos dois grupos. Conclusão: a hidrocinesioterapia praticada por gestantes saudáveis permitiu adequada adaptação hemodinâmica e metabólica materna, com melhor capacidade cardiovascular, desempenho físico materno no esforço submáximo e qualidade na composição e distribuição do peso corporal, sem prejuizo neonatal.

PALAVRAS-CHAVE: Capacidade cardiovascular; Composição corporal; Exercício aquático; Gravidez normal; Resultados perinatais

Resumo de Tese

\section{Fatores de risco, proteção e freqüência de perda de massa óssea em mulheres na pós-menopausa de uma Unidade Básica de Saúde da região Sul do Municipio de São Paulo-SP}

Risk and protection factors and bone mass loss frequency in postmenopausal women from a basic health care unit of the south region of São Paulo municipality

Autora: Sonia Raquel Wippich Coelho de Magalhães Leal

Orientador: Prof.Dr. João Carlos Mantese

Dissertação apresentada à Faculdade de Medicina da Universidade de Santo Amaro para obtenção do título de Mestre em Saúde Materno-infantil, em 12 de junho de 2005.

Objetivo: detectar fatores de risco e fatores de proteção para perda de massa óssea em mulheres na pós-menopausa em uma Unidade Básica da Região Sul do Município de São Paulo, correlacionando-os com os achados de densitometria óssea. Métodos: foram estudadas $69 \mathrm{mu}-$ lheres na pós-menopausa com idade igual ou superior a 50 anos. Responderam ao questionário do Programa de Atenção Integral à Saúde da Mulher (Secretaria de Estado da Saúde - Centro de Referência da Saúde da Mulher) onde estavam elencados todos os fatores que pudessem interferir com a perda de massa óssea. Divididas em 2 grupos de acordo com o diagnóstico da densitometria óssea: Normal GI $(n=16)$ e perda de massa óssea GII $(n=$ 53). Resultados: média de idade GI $=59,9$ GII $=61,0$; Proporção de perda de massa óssea $=53 / 69=0,76,81 \%$; Localização na coluna vertebral $n=22 / 41,5 \%$, no fêmur $n$ $=4 / 7,5 \%$, em ambos $\mathrm{n}=27 / 50,9 \%$; Idade da menarca GI
$=12,62, \mathrm{GII}=13,65 ;$ Idade da menopausa GI= 47,62, GII = 47,84; Tempo de menopausa GI = 12,03, GII = 13,0; Período do menacme $\mathrm{GI}=35$, GII $=34,8$; Paridade $\mathrm{GI}=4$, GII $=$ 3,9; Tempo de amamentação (meses) GI = 28,5, GII = 16,8; Peso GI $=84 \%$ GII $=69,8 \%$ em sobrepeso; IMC GI $=33$, $\mathrm{GII}=28 \mathrm{~kg} / \mathrm{m}^{2}$; Presença dos fatores de risco GI/GII: não-uso de TRH > GI. Presença de fatores de risco intragrupo GI e GII: Baixa escolaridade não uso de TRH e cor branca. Menor presença de fatores de proteção GI: Exercícios e em GII: Uso de contraceptivos. Conclusão: dentre os fatores de risco para perda de massa óssea encontrou-se a maior idade da menarca e dentre os fatores de proteção o peso e o IMC foram significantes.

PALAVRAS-CHAVE: Perda de massa óssea; Fatores de risco; Fatores de proteção 